\title{
Typ-2-Asthma: Die Perspektive der 1970er Jahre hinter uns lassen
}

\author{
Type-2 Asthma: Leaving Behind the Perspective \\ of the Seventies
}

\author{
Thomas Rothe und Tsogyal Daniela Latshang \\ Kantonsspital Graubünden, Chur
}

\begin{abstract}
Zusammenfassung: Die Diagnose «Asthma bronchiale» überspannt schirmartig verschiedene Phänotypen dieses Krankheitsbildes. Die Differenzierung dieser Phänotypen und ihrer Overlaps wird immer wichtiger, da heute Phänotyp-spezifische Behandlungsansätze existieren, die nicht bei jeder Asthmaform Wirkung zeigen. Dazu gehören die Strategie der gezielten Allergenkarenz, die Allergen-Immuntherapie und vor allem auch die neu zur Verfügung stehenden Biologika für Asthma. Behandelbare Krankheitsmuster, sogenannte «Treatable Traits», erfordern eine gezielte Diagnostik. Das notwenige Wissen, um diese Merkmale identifizieren zu können, muss sich in der Praxis noch etablieren.
\end{abstract}

Schlüsselwörter: Asthma, Bronchiale Hyperreaktivität, COPD, Asthma-Phänotypen, Eosinophilie

\begin{abstract}
The diagnosis 'bronchial asthma' spans different phenotypes of this disease like an umbrella. The differentiation of these phenotypes and their overlaps is becoming increasingly important, as the phenotype-specific treatment approaches of today are not effective with every form of asthma. These approaches include the strategy of allergen avoidance, allergen immunotherapy and, most importantly, the newly available biologics for asthma. Treatable disease patterns, so-called 'treatable traits', require targeted diagnostics. The knowledge necessary to identify these traits still needs to be established in practice.
\end{abstract}

Keywords: Asthma, bronchial hyperresponsiveness, COPD, asthma phenotypes, eosinophilia

Résumé: Le diagnostic «asthme bronchique» recouvre les différents phénotypes de ce type de maladie. La différenciation de ces phénotypes et de leurs chevauchements devient de plus en plus importante, car il existe aujourd'hui des approches thérapeutiques spécifiques aux phénotypes qui ne sont pas efficaces pour toutes les formes d'asthme. Il s'agit notamment de la stratégie d'évitement ciblé des allergènes, de l'immunothérapie allergénique et, surtout, des nouveaux médicaments biologiques disponibles pour l'asthme. Les schémas pathologiques traitables, appelés «traits traitables», nécessitent des diagnostics ciblés. Les connaissances nécessaires pour identifier ces traits doivent encore être établies dans la pratique.

Mots-Clés: Asthme, hyperréactivité bronchique, BPCO, phénotypes de l'asthme, éosinophilie

Obwohl das allergische Asthma bereits im 19. Jahrhundert wissenschaftlich beschrieben worden ist [1], galt Asthma oft noch lange als Nervenerkrankung (Asthma nervosa) und die Medizin nahm ausschliesslich die akuten Atemnotanfälle wahr, die - aufgrund des Fehlens wirksamer Medikamente - manchmal so eindrucksvoll wie ein epileptischer Krampfanfall anmuteten. Später erklärte F. Alexander [2] das Asthma zu einer der Krankheiten der psychosomatischen «Holy Seven». Ab den 1970er Jahren des vergangenen Jahrhunderts wurden die pathophysiologischen Grundlagen der allergischen Entzündung jedoch immer besser erforscht. Diese Erkenntnis führte zum Siegeszug inhalativer Kortikosteroide (ICS) in der Asthmatherapie. Allerdings zeigte sich dann in grossen Querschnittstudien, dass bei mehr als der Hälfte der Menschen mit Asthma die eosinophile Entzündung der Mukosa nicht als Reaktion auf ein typisches Inhalationsallergen zu interpretieren ist $[3,4]$ und die Symptome manchmal sogar ICS-resistent [5] sind. Gerade das schwere Asthma ist oft von einer hoch eosinophilen Entzündung gekennzeichnet, obwohl vielfach keine allergische Reaktion vorliegt [6].

Bis heute gehen aber noch viele Medizinschaffende davon aus, dass Asthma ein allergisches, TH2-vermitteltes Geschehen mit assoziierter bronchialer Hyperreaktivität (BHR) ist. Als alleinige Auslöser für akute Exazerbationen

$\begin{array}{ll}\text { Im Artikel verwendete Abkürzungen } \\ \text { ACC } & \text { Asthma-COPD-Koexistenz } \\ \text { BHR } & \text { Bronchiale Hyperreaktivität } \\ \text { COPD } & \text { Chronisch obstruktive Lungenerkrankung } \\ \text { FEV } & \text { Forciertes exspiratorisches Volumen in der 1. Sekunde } \\ \text { GINA } & \text { Global Initiative for Asthma } \\ \text { ICS } & \text { Inhalative Kortikosteroide } \\ \text { OCS } & \text { Orale Kortikosteroide }\end{array}$


werden Allergenkontakt und Virusinfekte mit Störung der Barrierefunktion des Epithels angesehen, sodass Allergene besser eindringen können, sowie die Exposition mit unspezifischen, irritativen Noxen wie Austrocknung der Atemwege durch kalte Luft (Anstrengungs-induzierter Bronchospasmus), Gase oder Rauch. Dieses klinische Verständnis trifft aber überwiegend nur auf Menschen mit allergischem «Early-onset»-Asthma zu. Selbst bei diesem sieht man jedoch gelegentlich im jahrzehntelangen Verlauf eine zunehmende Entkopplung der Symptomatik von exogen-allergischen Triggern, d.h. es findet sich eine eosinophile Entzündung trotz Allergenkarenz.

Bereits vor 103 Jahren formulierte Rackemann eine Beobachtung, die konträr zur späteren «70-Jahre-Perspektive» positioniert ist. Er beschrieb 150 Personen mit einem perennealen «Late-onset»-Asthma, entzündlicher Mitbeteiligung der oberen Atemwege und Nachweis einer ausgeprägten Bluteosinophilie, ohne dass Hinweise auf ein exogen-allergisches Geschehen zu finden waren. Dieser Phänotyp wurde von ihm «Intrinsic Asthma» genannt [7].

\section{Das eosinophile Adult-onset- Asthma}

Das Wissen, dass eine spezifische Allergenexposition zu perakuten Asthmaexazerbationen führen kann, ist alt. Vor 50 Jahren wurden dann zunehmend Studien bei Kindern mit allergischem Early-onset-Asthma unter Allergenkarenz-Bedingungen unternommen. In Misurina (I), Briançon (F) und Davos zeigte sich, dass Kinder mit Milbenasthma unter Allergenkarenz im Hochgebirge oft asymptomatisch wurden und dass mittels HistaminProvokation der Nachweis erbracht werden konnte, dass die BHR unter Allergenkarenz massiv regredient war [8].

Dazu kontrastiert aber das eosinophile Late-onset -Asthma, das, wie von F. M. Rackeman beschrieben, perenneal verläuft, ohne erkennbare exogene Auslöser. Auch heute noch sehen wir hier in der Therapie, dass sich nach dem Ende eines kurzzeitigen oralen Kortisonstosses in den Folgewochen spontan wieder eine zunehmende Blute- osinophilie entwickeln kann, mit Auftreten von produktivem Husten und Belastungsdyspnoe, was im Verlauf dann langsam zu einer erneuten Asthma-Exazerbation führt.

M. Turner-Warwick beschrieb vor mehr als 40 Jahren das Peak-flow-Muster des Morning-Dippings (Abb.1). So nannte sie die grosse Tagesvariabilität der Peak-flowWerte bei unkontrolliertem Asthma, die Ausdruck einer ausgeprägten BHR ist. Klinisch findet sich dieses Bild meist beim allergischen Early-onset-Asthma.

Turner Warwick wies aber auch auf ein zweites, von ihr Drifter-Typ (Abb. 2) genanntes Muster hin, bei dem es zu einer langsamen Verschlechterung der Peak-flow-Werte kommt ohne Zunahme der Peak-flow-Variabilität [9]. Hier steht nicht der Bronchospasmus im Vordergrund, sondern eine zunehmende Schwellung der bronchialen Mukosa sowie «Mucoid Impaction» der Bronchiolen mit Entwicklung einer auf Betastimulatoren nicht reversiblen Obstruktion.

Seit knapp 15 Jahren werden Asthma-Phänotypen nicht mehr auf rein empirischer Basis beschrieben, sondern wissenschaftlich in sogenannten Cluster-Analysen erforscht. Diese bestätigten die grosse Gruppe von Personen mit allergischem Early-onset-Asthma und konkordanter Entzündung. Gemeint ist damit, dass typische Asthmasymptome, die Ausdruck einer zunehmenden BHR sind, mit dem Ausmass der eosinophilen Entzündung korrelieren. Es fand sich allerdings auch ein Cluster mit sehr ausgeprägter Bluteosinophilie, aber weitgehendem Fehlen typischer Asthmasymptome, was auf eine geringere BHR schliessen lässt. Die Autoren wiesen bei diesem Cluster auf die Diskordanz von typischen Asthmasymptomen und Entzündung hin (Abb. 3) [10].

Dieses Cluster entspricht klinisch dem Drifter-Typ von Turner-Warwick wie auch dem Intrinsic Asthma von Rackemann. Dieser Phänotyp des eosinophilen Late-onset-Asthmas ist durch eine intrinsische eosinophile Entzündung gekennzeichnet, d.h. beim Unterschreiten einer individuell nötigen Schwellendosis von ICS und teilweise auch oralen Kortikosteroiden (OCS) entwickelt sich spontan wieder eine zunehmende Bluteosinophilie und konsekutiv eine erneute, langsam progrediente Asthma-Exazerbation. Dies erklärt, weshalb bei schweren Verläufen dieses Krankheitsbildes trotz ausgebauter inhalativer The-

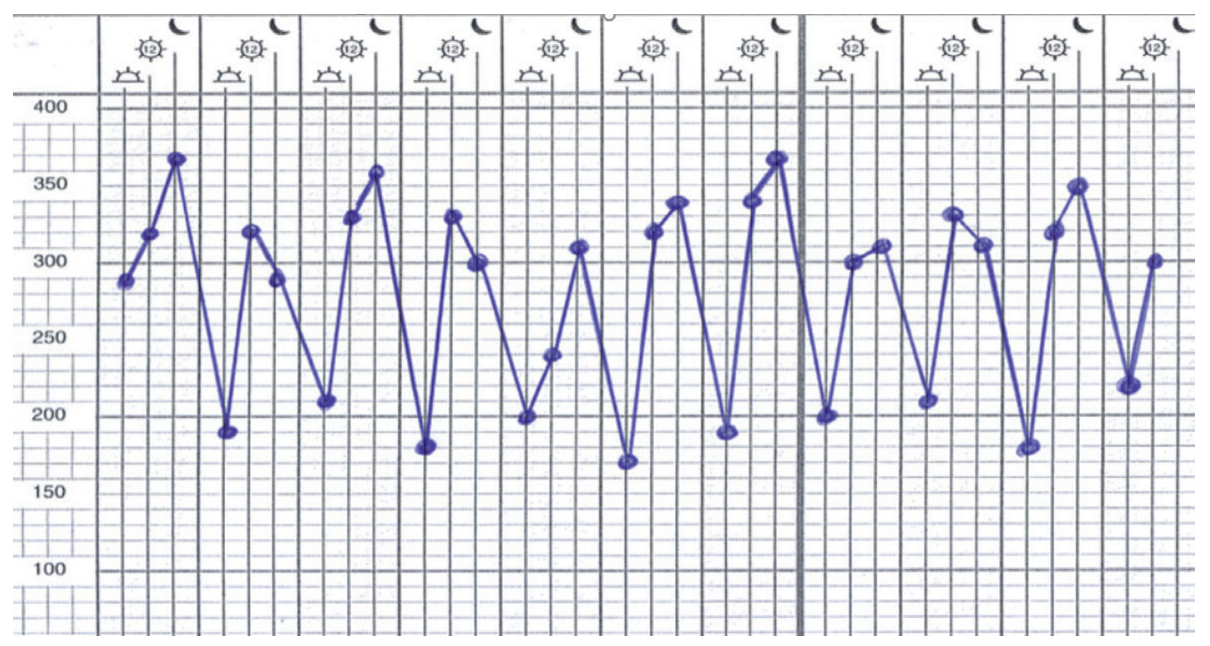

Abbildung 1. Peak-flow-Muster des Morning-Dippings [9]. 

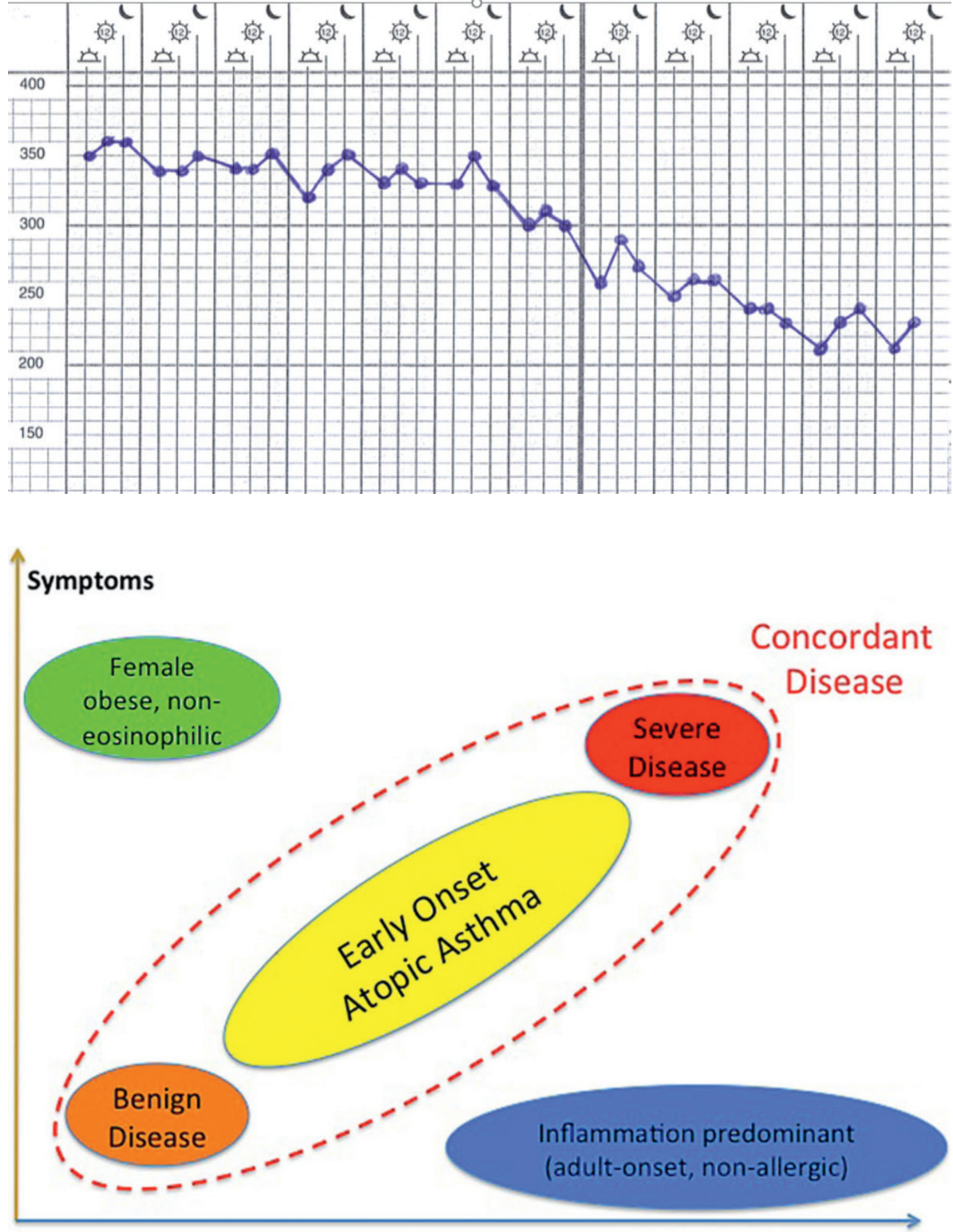

Eosinophilic Inflammation
Abbildung 2. Drifter-Typ [9].
Abbildung 3. Das adult-onset Asthma (inflammation predominant) zeigt trotz starker Ausprägung der eosinophilen Entzündung nur wenig typische Asthmasymptome [10]. rapie mehrmals jährlich Exazerbationen auftreten können [11]. Wurden dann früher täglich OCS in ausreichender Dosis appliziert, liessen sich diese spontanen Exazerbationen meist vermeiden, allerdings unter Inkaufnahme einer Steroidosteoporose. Heute stellen Asthma-Biologika in dieser Situation die bevorzugte Therapieoption dar [12].

Beim eosinophilen Adult-onset-Asthma werden Exazerbationen zwar auch durch virale Atemwegsinfekte ausgelöst, können aber wie beschrieben auch spontan auftreten. Wenn dies geschieht, kommt es aufgrund der entzündlichen (eosinophilen) Mitbeteiligung der oberen Atemwege (chronische Rhinosinusitis mit nasalen Polypen $=$ CRSwNP) $\mathrm{zu}$ vermehrter Rhinorrhoe, Hyposmie und produktivem Husten. Klinisch wird diese Symptomatik, sofern nicht zu diesem Zeitpunkt nach einer Bluteosinophilie gesucht worden ist, als Infektexazerbation missinterpretiert. Eine Eosinophilie gilt hier als signifikant, wenn im Differenzialblutbild mehr als 0,3 G/l eosinophile Granulozyten nachweisbar sind [9]. Leider gibt es in allge- mein-internistischen Praxen häufig Laborautomaten, die auch beim grossen Blutbild die genaue Anzahl Eosinophiler nicht separat angeben.

Die Schweizer SAPALDIA-Studie [2] konnte zeigen, dass ca. $30 \%$ der Bevölkerung auf mindestens eines der häufigsten lokalen Inhalationsallergene sensibilisiert sind. Bei nur ca. $50 \%$ der Betroffenen ist diese Sensibilisierung klinisch aktuell, d.h. natürlicher Allergenkontakt führt zu einer allergischen Reaktion. Bei den anderen ist sie aber nur latent, d.h. klinisch stumm. Entsprechend finden sich auch bei Personen mit eosinophilem Late-onset-Asthma Sensibilisierungen, die oft aber latent sind. Eine alleinige Pollensensibilisierung kann nicht die Kausalität eines perennealen Asthmas ohne saisonalen Peak beweisen. In manchen Fällen sind Sensibilisierungen sogar klinisch aktuell, erklären aber nicht die ganzjährige entzündliche Aktivität und die fehlende Besserung unter Allergenkarenz-Bedingungen [13]. Hier besteht dann ein Overlap von allergischem und nicht allergischem Asthma. Schon S. Wenzel wies darauf 


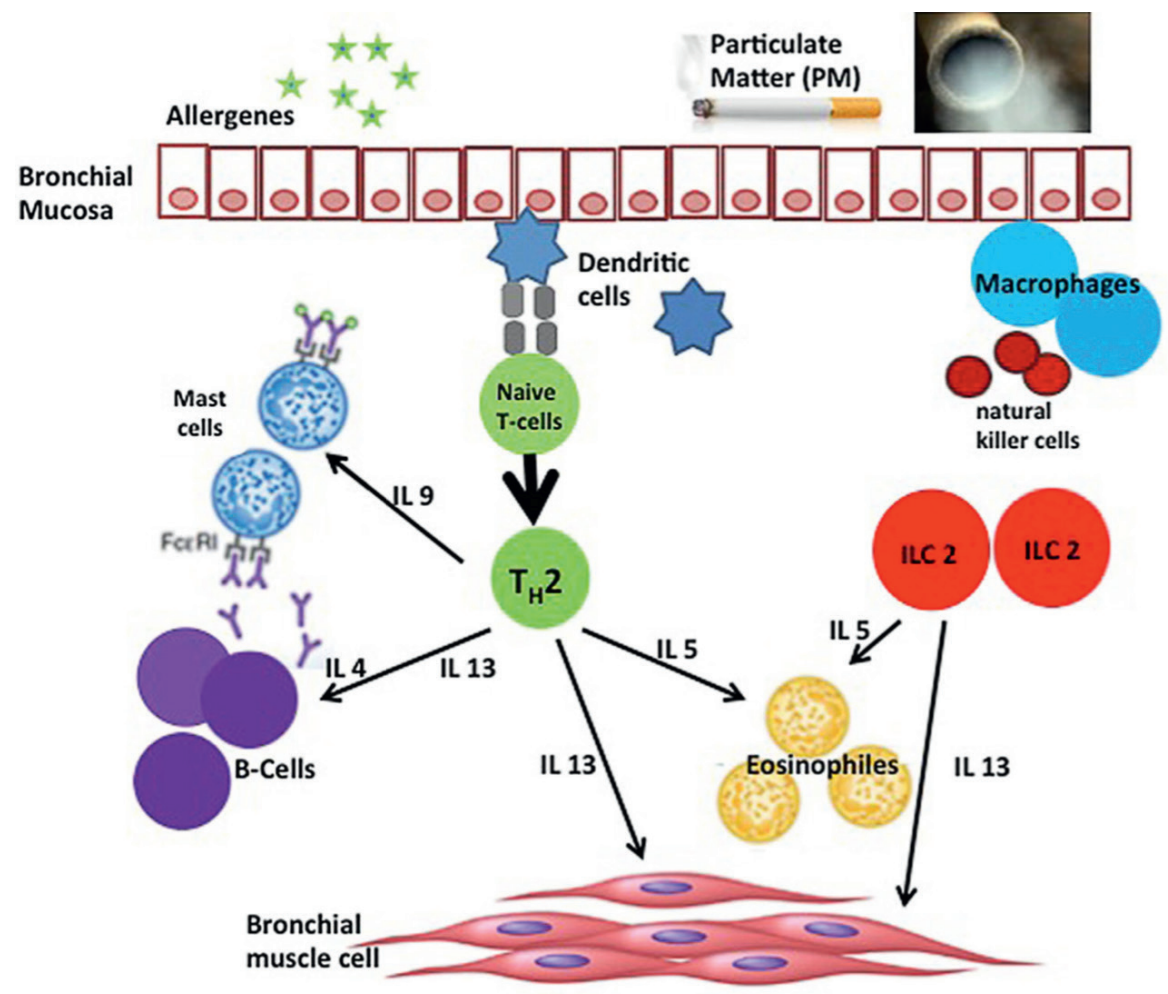

Allergic eosinophilic inflammation
Non allergic eosinophilic inflammation
Abbildung 4. Phänotyp-abhängige Bildung eosinophiler Granulozyten via TH2-Lymphozyt bei der Allergie versus ILC2 bei der nicht-allergischen eosinophilen Entzündung [15]. hin, dass nicht jedes Asthma, bei dem sich serologisch oder im kutanen Pricktest allergenspezifische IgE nachweisen lassen, als «allergisch» qualifiziert werden darf [14].

\section{Asthma-Phänotypen und ihre teils diametrale Klinik}

Dem Asthma liegt oft eine eosinophile Entzündung zugrunde, z.B. auf der Basis einer allergischen Reaktion, an der u.a. TH2-Lymphozyten beteiligt sind. Daneben, v.a. beim Adult-onset-Asthma, kann die eosinophile Entzündung jedoch auch über ILC (Innate Lymphocytic Cells) vermittelt werden. Als Auslöser wird hier eine Störung der Barrierefunktion des respiratorischen Epithels durch virale Infekte, Luftverschmutzung und das Rauchen angenommen (Abb. 4) [15]. Beide Phänotypen werden heute unter dem Begriff «Typ-2-Asthma» subsummiert.

Wie oben beschrieben differiert die Klinik der beiden Unterformen teilweise stark. Beim allergischen Early-onset-Asthma mit BHR kann es zu perakuten Exazerbationen kommen, oft aber mit gutem Ansprechen auf einen Bronchodilatator. Das eosinophile Adult-onset-Asthma ist eher durch eine sich langsam entwickelnde Obstruktion ohne nachhaltige Wirkung eines Relievers gekennzeichnet.

Bei Diagnosestellung eines Asthmas im Erwachsenenalter fällt es der Patientin/dem Patienten oft schwer, die Diagnose «Asthma» anzunehmen, da im Verständnis der meisten Menschen Asthma mit einem frühen Beginn und plötzlichen Asthmaanfällen als Reaktion auf exogene Allergene assoziiert ist. Entsprechend ist eine tiefergreifende Aufklärung notwendig. Die ätiologisch unklare, spontan rekurrierende Entzündung der Gelenksschleimhaut bei der rheumatoiden Polyarthritis kann hier als analoger Erklärungsversuch dienen.

Obwohl das eosinophile Adult-onset-Asthma ja bereits vor 103 Jahren erstmals beschrieben worden war, bestand in der ärztlichen Welt lange diesbezüglich ein Neglect. Asthma wurde bisher fast immer als eine allergische TH2Entzündung angesehen. Diese Perspektive der 1970er und 1980er Jahre ist interessanterweise u.a. von der Pharmaindustrie aufgeweicht worden. Seit 20 Jahren sind Anti-IL5-Antikörper beim Asthma in Erprobung [16] und Anti-IL5 bzw. Anti-IL4/13-Antikörper werden heute erfolgreich beim schweren eosinophilen Asthma verschrieben [17]. Erst als sich zeigte, dass diese praktisch nur beim hoch eosinophilen, meist nicht allergischen Adult-onset-Asthma wirksam sind, kam es auch im medizinischen Bewusstsein zur Rückbesinnung auf diesen nicht IgE-vermittelten Asthma-Phänotyp.

\section{Verwechslungsgefahr mit der COPD}

Selbst aktuelle Lehrbücher enthalten teilweise noch Tabellen mit Gegenüberstellung der Symptome und Befunde beim Asthma versus COPD. Hier wird hervorgehoben, dass sich Asthma meist in der Kindheit manifestiert, allergische Trigger aufweist, die bronchiale Obstruktion ein 

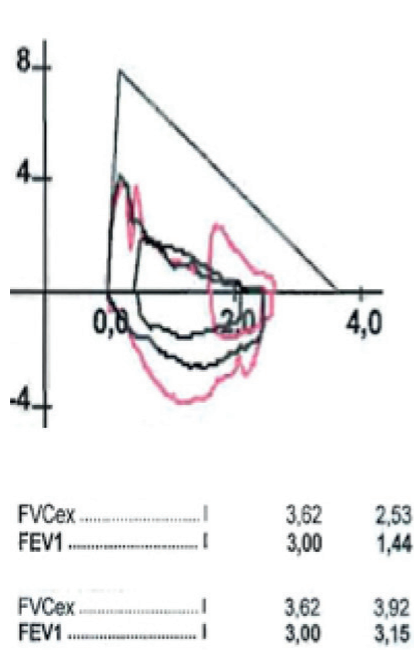

Abbildung 5. Spirometrie beim exazerbierten eosinophilen Adultonset Asthma mit fehlender Besserung nach Bronchospasmolyse (links) und Kontroll-Spirometrie (rechts) nach oralem Steroidstoss mit voller Reversibilität der Obstruktion durch die Kortikosteroide.

rasches und deutliches Ansprechen auf Betastimulatoren zeigt, Asthmatiker meist nicht rauchen bzw. geraucht haben usw.

All dies trifft nicht für den Phänotyp des eosinophilen Adult-onset-Asthmas zu. Diese Patientinnen und Patienten weisen überwiegend eine positive Nikotinanamnese auf, manifestieren die Symptomatik oft erst in der zweiten Lebenshälfte, und Allergien spielen hier nur eine untergeordnete Rolle.

Kommt es bei solchen Personen zu einer langsam progredienten Exazerbation, entwickelt sich eine Belastungsdyspnoe, kein Anstrengungs-induzierter Bronchospasmus! Erfolgt die medizinische Erstabklärung erst zu diesem Zeitpunkt, ergibt die Lungenfunktion eine auf Betastimulatoren nicht reversible Obstruktion, und die Nikotinanamnese ist in vielen Fällen positiv. Die Betroffenen erhalten in dieser Situation die Diagnose «COPD», werden mit einem oralen Steroidstoss und Antibiotika über fünf Tage behandelt und profitieren subjektiv davon sehr. Nach Absetzen der Steroidtherapie entwickeln sich aber im Verlauf oft immer wieder Exazerbationen mit erneuter Notwendigkeit o.g. medizinischer Interventionen.

Würde dagegen zum Zeitpunkt der Exazerbation, noch vor dem Beginn des kurzen Steroidstosses, ein Differenzial-Blutbild eine Bluteosinophilie objektivieren und vor wie nach dem Steroidstoss eine Spirometrie erfolgen, wäre erkennbar, dass sich die Lungenfunktion infolge der Steroidtherapie vorübergehend wieder normalisiert. Damit ist die Diagnose COPD nicht mehr haltbar und es sollte ein eosinophiles Adult-onset-Asthma diagnostiziert werden (Abb. 5).

Dieser Asthmaphänotyp kann also eine COPD imitieren, und er lässt sich nur dann diagnostizieren, wenn die Bluteosinophilen (alternativ das exhalatorisch gemessene
$\mathrm{NO}=\mathrm{FeNO}$ ) kontrolliert und eine Spirometrie mindestens nach jedem Steroidstoss durchgeführt werden. Schon 1958 wurde in der Literatur beschrieben, dass besonders erwachsene Asthmakranke mit Eosinophilie von einem oralen Kortisonstoss nachdrücklich profitieren [18].

In den nächsten Jahren werden neue Biologika fürs Asthma zugelassen werden. Vermutlich kommen dann auch neue Parameter hinzu, die präziser abschätzen lassen, wie suffizient die Wirkung dieser individualisierten Therapie beim einzelnen Individuum sein wird. Insofern ist es wichtig, dass Menschen mit schwerem Asthma regelmässig vom Spezialisten gesehen werden. Dieser kennt auch die Limitatio der einzelnen Biologika (SL-Liste) und ist nach Einholen der Kostengutsprache berechtigt, diese zu verordnen.

Über Jahre wurde in den COPD-GOLD- wie auch in den GINA-Asthmarichtlinien das Asthma-COPD-Overlap beschrieben. Seit zwei Jahren sprechen die Guidelines [19] nur noch von der möglichen Asthma-COPD-Koexistenz (ACC). Eine Form des ACC liegt vor, wenn eine Person mit Early-onset-Asthma in der Adoleszenz die Asthma-Symptomatik verliert, zu rauchen beginnt, naturgemäss dann mit grösserer Wahrscheinlichkeit eine COPD entwickelt [20], lebenslang aber noch Zeichen einer BHR aufweist, die einer Therapie mit ICS bedarf.

Von einer zweiten Form des ACC muss gesprochen werden, wenn eine vorbestehende stabile COPD im Verlauf durch die Neumanifestation einer zusätzlichen, eosinophilen asthmatischen Entzündung kompliziert wird, d.h. spontane Exazerbationen auslöst. Sie lässt sich nur durch den Nachweis der Eosinophilie und einer massiven Besserung der $\mathrm{FEV}_{1}$ unter einem systemischen Steroidstoss diagnostizieren.

Das Vorliegen eines Anteils fixierter bronchialer Obstruktion beim Asthmatiker bedeutet aber nicht zwingend das Vorliegen einer ACC. Kinder mit schwerem Asthma erreichen im Alter von 20 Jahren oft nicht eine normwertige $\mathrm{FEV}_{1}$ [21]. Der jährliche $\mathrm{FEV}_{1}$-Verlust muss aber später nicht zwangsläufig mehr als $50 \mathrm{ml}$ pro Jahr betragen, wenn die Asthmatherapie im Erwachsenenalter suffizient ausgestaltet werden kann. Vor allem beim eosinophilen Adultonset-Asthma kann sich auch erst im Erwachsenenalter durch bronchiale Umbauprozesse, die Airway Remodeling genannt werden, ein Anteil fixierter Obstruktion entwickeln Diese beiden Beispiele sollten nicht als COPD-Anteil beurteilt werden. Eine Person mit jahrzehntelangem schwerem Asthma, die ein Leben lang nicht geraucht hat, würde es nicht goutieren, wenn man ihr die Diagnose «COPD» verpasst. «Dr. Google» gäbe sofort Auskunft darüber, dass ärztlicherseits ein kausaler Nikotinkonsum vermutet wird.

Beim Adult-onset-Asthma und der ACC ist es wichtig, dass nach einem oralen Steroidstoss die individuell beste $\mathrm{FEV}_{1}$ identifiziert wird [22]. Damit kann schon in der Diagnoseliste dokumentiert werden, dass es sich um ein Asthma handelt, d.h. die Therapie einer Exazerbation so lange fortgeführt werden muss, bis wieder der alte Bestwert erreicht wird. Beim ACC wird in dieser Situation nicht die Normalisierung der $\mathrm{FEV}_{1}$ angestrebt, sondern die zuvor erreichte individuell beste $\mathrm{FEV}_{1}$. 
Bei der reinen COPD sind je nach Risikogruppe ICS kontraindiziert, da sie das Risiko der Pneumonie und der Osteoporose-Entwicklung signifikant erhöhen können. Liegt aber eine ACC vor, müssen ICS verabreicht werden [23], bei häufigen Exazerbationen und Bluteosinophilie von $>0,3 \mathrm{G} / 1$ teilweise auch OCS. Zeigt sich dieser Bedarf, steht auch hier aufgrund der zweiten Diagnose «Asthma» die Möglichkeit einer Therapie mit Biologika offen [24].

\section{Beurteilung der Asthmakontrolle beim eosinophilen Adult-onset- Asthma}

Früher basierte die Klassifikation des Schweregrads der Erkrankung auf dem Ausmass der Symptomatik und dem der bronchialen Obstruktion. Heute dagegen wird der Schweregrad durch die Medikamentendosis definiert, die täglich notwendig ist, um eine suffiziente Asthmakontrolle zu erreichen. Die Beurteilung der Asthmakontrolle erfolgt mit Hilfe der Antworten auf spezifische Fragen zur Asthma-Symptomatik [19]. Allerdings basieren diese Fragen auf den typischen Asthmasymptomen, die mit dem aktuellen Ausmass der BHR korrelieren. Dies trifft sehr gut die

\section{Key messages}

- Wird unter der Arbeitsdiagnose «Asthma bronchiale» mit einer Therapie mit ICS begonnen und diese erweist sich als wirksam, sollte unbedingt auch eine weitere Abklärung des Asthma-Phänotyps erfolgen.

- Verschiedene Merkmale des Krankheitsbildes (Treatable Traits) legen es nah, dass eine spezifische Therapie des entsprechenden Phänotyps wirksam sein wird, z.B. Allergenkarenzmassnahmen, Allergen-Immuntherapie, und beim hoch-eosinophilen schweren Asthma die Therapie mit modernen Asthma-Biologika.

- Zur Identifikation von Treatable Traits sind die Erfassung von Symptomen der oberen Atemwege, eine gezielte Allergiediagnostik, die Beurteilung, ob die nachgewiesenen Sensibilisierungen das Krankheitsbild unterhalten, sowie die Bestimmung der Bluteosinophilenzahl und die Kontrolle der Spirometrie notwendig.

- Für die Bestimmung der Eosinophilen reicht das grosse Blutbild in der Praxis meist nicht aus, da eine genaue Quantifizierung der eosinophilen Granulozyten notwendig ist.

- Spirometrien sollten auch in Exazerbationen sowie immer nach der Gabe eines oralen Kortisonstosses erfolgen, damit die individuell beste $\mathrm{FEV}_{1}$ ermittelt werden kann bzw. ersichtlich ist, ob sich eine bronchiale Obstruktion unter Intensivierung der Therapie teilweise oder ganz zum Verschwinden bringen lässt.

\section{Lernfragen}

1. Welche Antwort ist falsch?

a) Bei jeder Konsultation eines Asthmatikers wegen seiner Erkrankung sollte kurz die aktuelle Asthmakontrolle erfasst werden.

b) Hört man in der forcierten Exspiration über den Lungen auskultatorisch Giemen, ist ein Asthma bewiesen, d.h. auf eine Lungenfunktionskontrolle kann verzichtet werden.

c) Moderne Asthma-Biologika sind auf der Spezialitätenliste mit der Limitation versehen, dass es sich um ein schweres, eosinophiles Asthma handeln muss.

d) Der Nachweis des eosinophilen Asthmas gelingt, wenn sich im Differenzialblutbild mehr als 0,3 G/l Eosinophile nachweisen lassen.

e) Auch wenn unter optimaler Therapie beim Asthma eine relevante bronchiale Obstruktion persistiert, sollte nicht von einer COPD gesprochen werden, wenn anamnestisch inhalative Noxen wie ein langjähriger Nikotinkonsum fehlen.

\section{Welche Antworten sind richtig? (Mehrfachauswahl)}

a) Spirometer sollten heute in jeder Allgemeinpraxis vorhanden sein und eingesetzt werden, genauso wie die Möglichkeit, ein EKG zu schreiben.

b) Die Zahl der Eosinophilen wird in jedem Blutbildautomaten in der Allgemeinarztpraxis erfasst.

c) Der Schweregrad eines Asthmas wird heute nicht mehr über das Ausmasse der Symptomatik bestimmt, sondern über die GINA-Therapiestufe, die nötig ist, um eine ausreichende Asthmakontrolle erzielen zu können.

d) Bei jedem Raucher mit obstruktiver Ventilationsstörung liegt per Definition eine COPD vor.

e) Eine positive Reaktion im Allergie-Pricktest beweist, dass ein allergisches Asthma vorliegt, wenn klinisch von einem Asthma ausgegangen werden kann.

Situation beim allergischen Early-onset-Asthma. Vom Adult-onset-Asthma wissen wir aber, dass oft eine Diskordanz zwischen typischer Asthmasymptomatik und dem Ausmass der eosinophilen Entzündung vorliegt und die Klinik eine COPD imitieren kann.

Insofern stellt sich beim Adult-onset-Asthma die Frage, ob nicht hier die Art und Weise, wie nach GINA die Asthmakontrolle erfasst wird, durch das Hinzufügen der aktuellen spirometrischen Messwerte ergänzt werden müsste [25]. Die aktuelle $\mathrm{FEV}_{1}$ kann dann mit dem individuellen Bestwert verglichen werden. GINA sah dies bis vor wenigen Jahren noch selbst vor, liess dann aber den Parameter Spirometrie fallen. Vermutlich aber nur deshalb, weil sich GINA als globale Guideline versteht und der Aufwand der regelmässigen Messung einer Spirometrie die medizinischen Ressourcen ärmerer Länder überfordert hatte. 


\section{Zusammenfassung}

Asthma ist eine sehr häufige Erkrankung. Bei genauer Betrachtung muss dieser Begriff aber als ein Schirm angesehen werden, unter dem verschiedene Phänotypen mit teils ganz unterschiedlicher Klinik subsummiert sind. Da die Therapieoptionen in den letzten Jahren massiv zugenommen haben, jedoch teilweise nur Phänotyp-spezifisch Wirkung aufweisen, ist heute eine differenziertere Beschreibung des Krankheitsbildes notwendig. Eine wichtige Rolle spielen dabei das Alter der Erstmanifestation, der eventuelle Nachweis einer Bluteosinophilie, das Vorhandensein von Sensibilisierungen gegen typische Inhalationsallergene und die Beurteilung, ob diese Sensibilisierungen klinische Aktualität besitzen oder ob sie nur klinisch latent sind.

\section{Bibliografie}

1. Blackley $\mathrm{CH}$. Experimental researches on the causes and nature of catarrhus aestivus (hay fever or hay asthma). London; Baillière Tindall \& Cox: 1873.

2. Alexander F. Psychosomatic medicine. Its principles and applications. Berlin; De Gruyter: 1951.

3. Wüthrich B, Schmid Grendelmeier P, Schindler C, et al. Prevalence of atopy and respiratory allergic diseases in the elderly SAPALDIA population. Int Arch Allergy Immunol. 2013;162: $143-148$.

4. Arbes Jr SJ, Gergen PJ, Zeldin DC. Asthma cases attributable to atopy: results from the Third National Health and Nutrition Examination Survey. J Allergy Clin Immunol. 2007;120:11391145.

5. Peters, MC, Kerr S, Dunican EM, et al. Refractory airway type 2 inflammation in a large subgroup of asthmatic patients treated with inhaled corticosteroids. J Allergy Clin Immunol. 2017; 143:104-111.

6. Amelink M, de Groot JC, de Nijs SB, et al. Severe adult-onset asthma: A distinct phenotype. J Allergy Clin Immunol. 2013; 132:336-341

7. Rackemann FM. A Clinical study of one hundred and fifty cases of bronchial asthma. Arch Int Med. 1918;22:517-523.

8. Peroni DG, Boner AL, Vallone G, Antolini I, Warner JO. Effective allergen avoidance at high altitude reduces allergen-induced bronchial hyperresponsiveness. Am J Resp Crit Care Med. 1994;149:1442-1446.

9. Turner-Warwick M. On observing patterns of airway obstruction in chronic asthma. Br J Dis Chest. 1977;71:73-86.

10. Haldar P, Pavord ID, Shaw DE, et al. Cluster analysis and clinical asthma phenotypes. Am J Respir Crit Care Med. 2008;178: 218-224.

11. Chung KF, Wenzel SE, Brozek L, et al. International ERS/ATS guidelines on definition, evaluation and treatment of severe asthma. Eur Respir J. 2014;43:343-373.
12. Holguin F, Cardet JC, Chung KF, et al. Management of severe asthma: a ERS/ATS guideline. Eur Respir J. 2020;55:1900588, DOI 10.1183/13993003.00588-2019.

13. Rothe T. Allergy and asthma exacerbation: Adding to the Pro/ Con debate in this journal. Respirology. 2011:16,378-379.

14. Wenzel S. Defining of the persistent adult phenotypes. Lancet. 2006;368:804-813.

15. Brusselle G, Maes T, Bracke K. Eosinophils in the spotlight: eosinophilic airway inflammation in nonallergic asthma. Nat Med. 2013;19:977-981.

16. Hart TK, Cook RM, Zia-Amirhosseini P, et al. Preclinical efficacy and safety of mepolizumab (SB-240563), a humanized monoclonal antibody to IL-5, in cynomolgus monkeys. J Allergy Clin Immunol. 2001;108:250-257.

17. Peters MC, Wenzel SE. Intersection of biology and therapeutics: type 2 targeted therapeutics for adult asthma. Lancet. 2020;395:371-383.

18. Brown HM. Treatment of chronic asthma with prednisolone: significance of eosinphils in the Sputum. Lancet. 1958;2:12451247.

19. Global Initiative for Asthma. www.ginasthma.org; letzter Zugriff: 10.05.2021

20. Brutsche MH, Downs SH, Schindler C \& SAPALDIA Team. Bronchial hyperresponsiveness and the development of asthma and COPD in asymptomatic individuals: SAPALDIA cohort study. Thorax. 2006;61:671-677.

21. Lange P, Celli B, Agusti A, et al. Lung-Function trajectories leading to COPD. N Engl J Med. 2015;373:111-122.

22. Woolcock A, Rubinfeld AR, Seale JP et al. Thoracic society of Australia and New Zealand. Asthma management plan. Med J Aust. 1989; 151: 650-653.

23. Chalmers JD, Laska IF, Franssen FME, et al. Withdrawal of inhaled corticosteroids in COPD: a European Respiratory Society Guideline. Eur Respir J. 2020;55:2000351.

24. Pavord ID, Chanez P, Criner GJ, et al. Mepolizumab for eosinophilic COPD. N Engl J Med. 2017:377:1613-1629.

25. Rothe T. Asthma control measures reconsidered. Respirology. 2021;26:21-22.

\section{Historie}

Manuskript eingereicht: 30.05.2021

Nach Revision angenommen: 06.07.2021

\section{Interessenskonflikte}

Es bestehen keine Interessenskonflikte.

\section{Dr. med. Thomas Rothe}

Ltd. Arzt Pneumologie

Spital Davos und Kantonsspital Graubünden

Loestrasse 150

7000 Chur

thomas.rothe@ksgr.ch

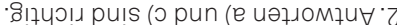
['70M7U

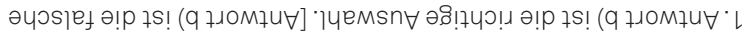

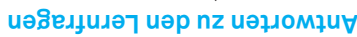

